\title{
Left-Behind Children in Parental Migration Are Susceptible To Cardiovascular Disease
}

\author{
Jinkui Lu ${ }^{1, *}$, Xiaojian Yin ${ }^{1}$, Lihong Mao² \\ ${ }^{1}$ Key Laboratory of Adolescent Health Assessment and Exercise Intervention, Ministry of Education, School of Physical Education and \\ Health, East China Normal University, Shanghai, China \\ ${ }^{2}$ Department of Physical Education, Jingganshan University, Ji'an, China \\ *Corresponding author: jinkui_lu@163.com
}

Received February 02, 2014; Revised February 18, 2014; Accepted March 22, 2014

\begin{abstract}
Objective: Left-behind children are rural children below 18 years of age who are left at home by their migrating parents. Although developmental, emotional and social problems have been reported on these left-behind children, No studies to date have been performed to examine the susceptibility of these left-behind children to cardiovascular disease. Methods: 2457 rural children, among whom 1212 are left-behind children by their migrant peasant worker parents and 1245 are not left-behind children as a control group, were recruited to examine the characteristics of left-behind children for their differences in heart rate and blood pressure from not left-behind children. Results: The heart rate and blood pressure of the left-behind children were significantly higher than those in the control not left-behind children. Conclusions: Childhood loneliness, dissatisfaction and anxiety may augment the burden or load on the cardiovascular system of the left-behind children, which may increase their susceptibility to development of cardiovascular disease in later life.
\end{abstract}

Keywords: left-behind children, parental migration, cardiac vascular diseases

Cite This Article: Jinkui Lu, Xiaojian Yin, and Lihong Mao, "Left-Behind Children in Parental Migration Are Susceptible To Cardiovascular Disease.” Journal of Food and Nutrition Research, vol. 2, no. 3 (2014): 96100. doi: 10.12691/jfnr-2-3-1.

\section{Introduction}

Economic development has promoted rapid urbanization in China, resulting in the migration of a large number of rural residents to large cities for employment. The children of these migrant peasant workers have been kept at home in rural areas to be left-behind (China Women's Federation 2008). Recent national surveys have revealed that left-behind Chinese children have exceeded 58 million, accounting for over $25 \%$ of rural children. This number appeared to increase 3-fold since 2000, when the number was estimated 20 million (China Women's Federation 2008; Stocktaking Report 2011). Left-behind children, who suffered from early separation from their migrant peasant worker parents, have attracted attention from researchers in various fields (Fan et al. 2009; Li et al. 2010).

Left-behind children may encounter various difficulties due to the absence of their parents. Most studies have suggested that left-behind children are likely to suffer from a broad range of mental health problems (Fan \& Sang 2005; Wen \& Lin 2012), such as depression, anxiety and loneliness (Magwaza 1994; Hugo 2002; AguileraGuzman et al. 2004; Jones et al. 2004; Pottinger 2005; Ren \& Shen 2008; Fan et al. 2009), and may have low levels of satisfaction and happiness (Goodman 1997; Fan et al. 2009; Fan \& Zhao 2010; Gao 2010; Gao et al. 2010; Liu \& Ouyang 2010), poor academic performance (Wu et al. 2004; Amuedo-Dorantes \& Pozo 2010), and problematic behavior (Fan \& Sang 2005; Fan et al. 2009; Fan \& Fang 2010). However, the relationship between left-behind from parental migration and health is complex and dynamic. Besides mental health, parental migration may also affect the physical health and well-being of the left-behind children (Carballo et al. 1998), although studies on these issues are scarce. The impact of parental migration on the physical health of the left-behind children is an almost unexplored topic in China. Since China has experienced a larger number of left-behind children due to continuous urbanization, the country needs to develop effective policies to control the negative effects on both mental and physical health of the left-behind children.

Here we examined whether the negative mental health conditions, such as depression, anxiety and loneliness, found in left-behind children, may have an effect on their heart rate and blood pressure, which are easily affected by mental disorders and are important parameters to decide the well-being and the function of the cardiovascular system. Our results showed that the left-behind children have significantly greater levels of heart rate and blood glucose, suggesting that parental migration and the separation of children from their parents may increase the susceptibility of the left-behind children to development of cardiovascular diseases in later life.

\section{Materials \& Methods}




\subsection{Eligibility Criteria}

The research plan was approved by the Ethical Committee of the Graduate School of Health and Sport Sciences in East China Normal University.

The sample in the current study, including both leftbehind children and comparison children, was recruited from the rural counties of 28 provinces in China, covering more than $90 \%$ of the country are, and a population of $9 \times 10^{8}$. Among all investigations, we selected 2457 to be used in the current study, including 1212 left-behind children and 1245 children without parental migration as a control. The eligibility criteria for left-behind children are: [1] they were born and raised in the countryside; [2] All are aged 6 to 12; [3] One or both of their parents have migrated to cities for employment for at least two years. That is, all left-behind children had been separated from their patients for at least 2 years. Comparison children were recruited from the same schools where left-behind children were recruited. In the same school, the number of the samples used for this study was decided by the smaller number between the left-behind children and the controls, and samples used for study from the group of a greater number were randomly selected using lottery method. For example, if in one school, there were 45 qualified leftbehind children and 211 not left-behind children. We just included all 45 qualified left-behind children and used lottery method to select 45 not left-behind children from all 211. If in one school, there were 145 qualified leftbehind children and 71 not left-behind children. We thus included all 71 not left-behind children and used lottery method to select 71 from all 145 qualified left-behind children. This strategy aimed to balance the number from two groups and minimize the effect of the area and age distribution on the results. Therefore, our study has a comparable area and age distribution between the leftbehind group and control group. In the current study, we did not discriminate the difference of the left-behind children with one of the parent migration from those with both.

\subsection{Measurements}

\subsubsection{Demographic Characteristics}

All participants were asked to provide information on their gender, age, family economic status, food intake, heart rate and blood pressure. Children with congenital cardiac diseases were excluded from the current study. The parents have been adequately informed and have sent their approval to allow their children to take this survey. The survey has been fulfilled with the help of the parents and a Giving of Content form has been signed by the parents.

\subsubsection{Characteristics of Left-Behind Children}

The left-behind children were asked to answer several questions regarding their experience with parental migration. Sample questions included 'Who is migrating in your family?'; 'How old were you when your parent(s) migrated?'; 'How long has it been since your parent(s) migrated?'; 'How often does your migrating parent(s) come home?'; 'How often does your migrating parent(s) contact you?'; 'Who is taking care of you now?'; 'What is the educational level of the person who is primarily taking care of you?'.

\subsubsection{Life Satisfaction and School Satisfaction}

Life and school satisfaction were evaluated by two questions: 'How satisfied are you feeling with your life?' and 'How satisfied are you feeling with your schooling?' Both questions have a 5 -point scale $(1$ = very dissatisfied to 5 = very satisfied).

\subsubsection{Loneliness}

Loneliness was evaluated using the children's loneliness scale (Asher et al. 1984). The scale applies 16 items for assessing children's perceived loneliness and social dissatisfaction. The items are based on a 5-point Likert scale ranging from 1 (not at all true) to 5 (always true), with a higher score indicating a higher level of loneliness. Dr. Lu performed the Chinese translation of the children's loneliness scale, to make it easily understandable and applicable to the Chinese elderly in the present study. He is the guarantee of the validity of the content.

\subsubsection{Happiness}

The short version of Oxford Happiness Questionnaire (Hills \& Argyle 2002) was used (eight items) to measure the level of happiness perceived by the participants. The items are based on a 6 -point Likert scale ranging from 1 (not at all true) to 6 (always true). A higher score indicates a higher level of happiness perceived by the participants. Dr. Lu performed the Chinese translation of the Oxford Happiness Questionnaire, to make it easily understandable and applicable to the Chinese elderly in the present study. He is the guarantee of the validity of the content.

\subsubsection{Heart Rate and Blood Pressure Measurement}

Heart rate and blood pressure data were obtained from the most recent examination of the children at their family doctor's clinic, or at public hospitals, to assure the accuracy and reliability of the results.

\subsection{Data Analysis}

A chi-squared test (for categorical variables) or ANOVA (for continuous variables) was employed to examine the differences in individual demographic characteristics among the left-behind and not left-behind children. ANOVA was performed to compare the group differences in terms of satisfaction, loneliness, happiness, heart rate and blood pressure. Finally, a correlation study was applied to assess the correlation of mental dissatisfaction with heart rate or blood pressure. All statistical analyses were performed using Spss for Windows 21.0.

\section{Results}

\subsection{Sample Characteristics}

The characteristics of the participants are shown in Table 1. Among the 2457 participants, 1212 (49.3\%) were left-behind children with one or both parents migrating, while 1245 (50.7\%) were children living in the families without parental migration. There were no significant 
differences between the male-to-female ratios among the two groups. The mean age was 10.3 years old for the entire sample, with the children with migrating parents averaging 10.45 years old and the children without migrating parents averaging 10.12 years old. No significant differences were observed among the two groups in terms of the area and age distribution. There is no difference in the maternal and paternal education levels among the two groups. There are significant differences in the education levels of the primary caretaker of the children. No significant differences were detected among the groups in terms of family economic status.

Table 1. Demographic characteristics of the sample

\begin{tabular}{|c|c|c|c|c|}
\hline & Total & $\begin{array}{l}\text { Children with parental } \\
\text { migration }\end{array}$ & $\begin{array}{l}\text { Children without parental } \\
\text { migration }\end{array}$ & P-value \\
\hline$n$ & $2457(100 \%)$ & $1212(49.3 \%)$ & $1245(50.7 \%)$ & 0.56 \\
\hline \multicolumn{5}{|l|}{ Gender } \\
\hline Male & $1262(51.4 \%)$ & $623(49.4 \%)$ & $639(50.6 \%)$ & 0.82 \\
\hline Female & $1195(48.6 \%)$ & $589(49.3 \%)$ & $606(50.7 \%)$ & \\
\hline Age $(\mathrm{M} \pm \mathrm{SD})$ & $10.30 \pm 1.69$ & $10.45 \pm 1.89$ & $10.12 \pm 1.53$ & 0.08 \\
\hline Father's educational level & & & & 0.22 \\
\hline Primary school or lower & 968 (39.4\%) & 477 (39.4\%) & $491(39.4 \%)$ & \\
\hline Middle school & $1180(48.0 \%)$ & $578(47.7 \%)$ & $602(48.4 \%)$ & \\
\hline High school or higher & $309(12.6 \%)$ & $157(12.9 \%)$ & $152(12.2 \%)$ & \\
\hline Mother's educational level & & & & 0.15 \\
\hline Primary school or lower & $1013(50.0 \%)$ & $561(46.3 \%)$ & $584(46.9 \%)$ & \\
\hline Middle school & $465(42.2 \%)$ & $541(44.6 \%)$ & $552(44.3 \%)$ & \\
\hline High school or higher & $86(7.8 \%)$ & $110(9.1 \%)$ & $109(8.8 \%)$ & \\
\hline $\begin{array}{c}\text { Primary caretaker's } \\
\text { educational level }\end{array}$ & & & & $<0.0001$ \\
\hline Primary school or lower & & $887(73.2 \%)$ & $394(31.6 \%)$ & \\
\hline Middle school & & $303(25.0 \%)$ & $679(54.5 \%)$ & \\
\hline High school or higher & & $32(1.8 \%)$ & $172(13.9 \%)$ & \\
\hline Economic score $(\mathrm{M} \pm \mathrm{SD})$ & & $2.57 \pm 0.59$ & $2.48 \pm 0.48$ & 0.21 \\
\hline
\end{tabular}

Table 2. Differences in terms of satisfaction, loneliness and happiness

\begin{tabular}{|c|c|c|}
\hline Satisfaction Scores & Children with parental migration & Children without parental migration \\
\hline Life satisfaction $(\mathrm{M} \pm \mathrm{SD})$ & $3.12 \pm 0.63$ & $3.77 \pm 0.55$ \\
\hline Academic satisfaction $(\mathrm{M} \pm \mathrm{SD})$ & $2.95 \pm 0.52$ & $3.35 \pm 0.47$ \\
\hline Loneliness $(\mathrm{M} \pm \mathrm{SD})$ & $3.71 \pm 0.36$ & $<.001$ \\
\hline Happiness $(\mathrm{M} \pm \mathrm{SD})$ & $3.56 \pm 0.71$ & $<0.001$ \\
\hline
\end{tabular}

\subsection{Relationship of Mental Disorder With Parental Migration}

The differences in life satisfaction, loneliness and happiness were compared between two groups with/without parental migration and are shown in Table 2.
Parental migration was found to be significantly associated with life satisfaction, loneliness and happiness. Left-behind Children appeared to experience a lower degree of life satisfaction and happiness, and a higher degree of loneliness than the comparison children.

Table 3. Differences in terms of heart rate and blood pressure

\begin{tabular}{|c|c|c|}
\hline Satisfaction Scores & Children with parental migration & Children without parental migration \\
\hline Heart rate (rpm, $\mathrm{M} \pm \mathrm{SD}$ ) & $84 \pm 22$ & $79 \pm 18$ \\
\hline Systolic pressure (mmHg, $\mathrm{M} \pm \mathrm{SD}$ ) & $123 \pm 39$ & $115 \pm 28$ \\
\hline Diastolic pressure (mmHg, $\mathrm{M} \pm \mathrm{SD}$ ) & $82 \pm 18$ & $<0.01$ \\
\hline
\end{tabular}

\subsection{Relationship of Children's Heart Rate and Blood Pressure to Parental Migration}

Since childhood unhappiness, loneliness and anxiety may affect the proper development and function of children's cardiovascular systems, we aimed to investigate whether left-behind children may have significantly different heart rates and blood pressures than children who were not left-behind. We chose to examine heart rate and blood pressure, since these two parameters are relatively easier to obtain and are good indicates for heart development and function. We found that the left-behind children had a significantly higher heart rate, and significantly greater systolic and diastolic pressures (Table 3). Moreover, correlation tests showed that there is a strong correction between the life satisfaction, academic satisfaction, loneliness and happiness scores, and the heart rate and blood pressure in left-behind children (Table 4). These data suggest that childhood loneliness, dissatisfaction and anxiety of left-behind children may increase their susceptibility to development of cardiovascular diseases in later life.

Table 4. correlations between the life satisfaction, academic satisfaction, loneliness and happiness scores, and the heart rate and blood pressure in left-behind children

\begin{tabular}{|c|c|c|c|c|}
\hline Correlation $\left(\gamma^{2}\right)$ & Life satisfaction & Academic satisfaction & Loneliness & Happiness \\
\hline Heart rate & 0.89 & 0.68 & 0.88 & 0.92 \\
\hline Systolic pressure & 0.86 & 0.72 & 0.93 & 0.88 \\
\hline Diastolic pressure & 0.84 & 0.74 & 0.86 & 0.94 \\
\hline
\end{tabular}




\section{Discussion}

\subsection{Main Finding(s) of the Study}

Here, we compared childhood health outcomes related to parental migration in an extremely large and welldistributed sample. The selection criteria were strict and designed to exclude the confounding effects of age, area and sex distribution on the interpretation of our results.

The most important finding came from the second part of our study, in which we examine the heart rate and blood pressure in left-behind children. It is well-known that long-term mental discomfort may lead to physical disorder, especially susceptibility to cardiovascular diseases. Here we chose to examine heart rate and blood pressure, since these two parameters are relatively easier to obtain through questionnaires and are good indicators of heart development and function. Not only did we find a significantly higher heart rate and significantly greater systolic and diastolic pressures in those left-behind children, but we also found a strong correction between the life satisfaction, academic satisfaction, loneliness and happiness scores, and the heart rate and blood pressure in left-behind children. Our data, as far as we know, constitute the first study to show that parental migration not only results in mental discomfort like childhood loneliness, dissatisfaction and anxiety in left-behind children, but also may increase their susceptibility to development of cardiovascular diseases in later life.

\subsection{Comparison of The Study Findings with Results of the Other Similar International and National Studies}

Our data on the relationship of parental migration and childhood mental issues are consistent with most previous studies, showing that left-behind children have significantly low degrees of life satisfaction, academic satisfaction and happiness, along with higher degrees of loneliness (Amuedo-Dorantes \& Pozo 2010; AguileraGuzman et al. 2004; Fan et al. 2009; Fan \& Sang 2005; Fan \& Fang 2010; Fan \& Zhao 2010; Goodman 1997; Gao 2010; Gao et al. 2010; Hugo 2002; Jones et al. 2004; Liu \& Ouyang 2010; Magwaza 1994; Pottinger 2005; Ren \& Shen 2008; Wen \& Lin 2012; Wu et al. 2004). Nevertheless, the issue whether left-behind may affect the physical development and the health of the children has not been addressed before.

\subsection{Limitations of the Study}

Our study has several strengths, including a relative large number of samples (2457), a well-distribution of the parameters of the study and control groups, and a strictly random and blind way of collection of the data. However, the limitations of the current study should also be recognized. First of all, it is a cross-sectional study, since all the factors were measured at a single point in this study. Therefore, the analysis can only provide evidence of statistical association between those variables and the mental health status of the children. Secondly, since some of the information was gathered from the parents or guardians, which may have resulted in some reporting bias, such as under-reporting of stigmatizing behaviors or overreporting healthy behavior.

\subsection{A Bring to Home Message for Other Researchers and Policy Makers}

Serious attention should be paid to effects of parental migration on the health of left-behind children, in order to provide a better societal environment to protect them from both mental and physical disorders in later life.

\section{Acknowledgements}

The authors would like to thank LQ Jia, B Lu, RF Wu, CC Zhang, JH Wu, Q Guo, JJ Liu (Graduate School of Physical Education and Health, East China Normal University, Shanghai, China) for their contributions in the cohort study.

\section{References}

[1] Aguilera-Guzman RM, de Snyder VN, Romero M, Medina-Mora ME. Paternal absence and international migration: stressors and compensators associated with the mental health of mexican teenagers of rural origin. Adolescence. 2004; 39 (156): 711-23.

[2] Amuedo-Dorantes, C. \& Pozo, S. (2010) Accounting for remittance and migration effect on children's schooling. World Development, 38, 1747-1759.

[3] Asher, S. R. , Hymel, S. \& Renshaw, P. D. (1984) Loneliness in children. Child Development, 55, 1456-1464.

[4] Carballo M, Divino JJ, Zeric D. Migration and health in the european union. Tropical Med Int Health. 1998; 3 (12): 936-44.

[5] China Women's Federation (2008) National survey of left-behind children in rural areas. Available at: http://www.china.com.cn/zhibo/200802/27/content_10180165.htm (last accessed 1 November 2010).

[6] Fan, F. \& Sang, B. (2005) Absence of parental upbringing and liushou children's personality, academic achievements as well as behavior problems. Psychological Science, 28, 855-858.

[7] Fan, L. \& Zhao, W. (2010) A research of life satisfaction among rural left-behind children. Education, (2), 42-44.

[8] Fan, X. \& Fang, X. (2010) A comparison of problem behaviors between parent-present children and parent-absent children in different care-taking modes. Chinese Journal of Clinical Psychology, 18, 232-237.

[9] Fan, X., Liu, Q. \& Liu, Y. (2009) A social adaptation comparison of migrant children, rear children, and ordinary children. Journal of Beijing Normal University (Social Sciences), 215, 33-40.

[10] Gao, J. (2010) Analysis on the self-consciousness of rural migrant workers' left-behind children and the influencing factors. China Journal of Health Psychology, 18, 58-62.

[11] Gao Y, Li LP, Kim JH, Congdon N, Lau J, Griffiths S. The impact of parental migration on health status and health behaviours among left behind adolescent school children in China. BMC Public Health. 2010; 10: 56.

[12] Goodman R. The Strengths and Difficulties Questionnaire: a research note. J Child Psychol Psychiatry. 1997; 38 (5): 581-6.

[13] Hills, P. \& Argyle, M. (2002) The Oxford Happiness Questionnaire: a compact scale for the measurement of psychological well-being. Personality and Individual Differences, 33, 1073-1082.

[14] Hugo G. Effects of international migration on the family in indonesia. Asian Pac Migr J. 2002; 11 (1): 13-46.

[15] Jones A, Sharpe J, Sogren M. Children's experiences of separation from parents as a consequence of migration. Caribb J Soc Work. 2004; 3: 89-109.

[16] Li, X., Yuan, J., Gao, W., Luo, J. \& Du, Y. (2010) Research on negative emotions, behavior, personal relationship caused by leftbehind experience of rural children after growing up. China Journal of Health Psychology, 18, 92-94. 
[17] Magwaza, A. S. (1994) Migration and psychological status in South African black migrant children. The Journal of Genetic Psychology, 155, 283-291.

[18] Pottinger AM. Children's experience of loss by parental migration in inner-city Jamaica. Am J Orthopsychiatry. 2005; 75 (4): 485-96.

[19] Ren, N. \& Shen, L. (2008) A survey on loneliness status of children left in rural areas in primary school. China Journal of Health Psychology, 16, 754-756.
[20] Stocktaking Report (2011) Child Welfare in China. Available at: http://uploads/soft/1_110531163254.pdf (last accessed 2 July 2011).

[21] Wen, M. \& Lin, D. (2012) Child development in rural China: children left behind by their migrant parents and children of nonmigrant families. Child Development, 83, 120-136.

[22] Wu, N., Ding, J. \& Tang, Y. (2004) A survey report on the education of hometown-remaining children in rural areas. Educational Research, 297, 15-18, 53. 